\title{
Study on buoyancy convection phenomenon in the crystal growth process
}

\author{
DUAN Li \& KANG Qi \\ National Microgravity Laboratory, Institute of Mechanics, Chinese Academy of Sciences, Beijing 100190, China.
}

Real-time phase shift Mach-Zehnder interference technique, imaging technique, and computer image processing technique were combined to perform a real-time diagnosis of $\mathrm{NaClO}_{3}$ crystal, which described both the dissolution process and the crystallization process of the $\mathrm{NaClO}_{3}$ crystal in real-time condition. The dissolution fringes and the growth fringes in the process were obtained. Moreover, a distribution of concentration field in this process was obtained by inversion calculation. Finally, the buoyancy convection phenomenon caused by gravity in the crystal growth process was analyzed. The results showed that this convection phenomenon directly influences the growth rate of each crystal face in the crystal.

crystal growth, interferometry, buoyancy convection

\section{Introduction}

The growth process of crystal is a transportation process with interactions among heat, quality and momentum, which influences the crystal growth rate and controls the growth interface stability. As a result, this process influences the crystal growth quality. From this point, we can know that the fluid flow, the character of heat transfer and mass transfer have much close relation with the microstructure of growth crystal. The concentration field keeps changing inevitably during the growth process of solution crystal. Therefore, under the action of gravity, the buoyancy convection caused by the change of fluid concentration is also inevitable. On the other hand, the buoyancy convection changes the concentration distribution of the fluid, thus affects the transporting quality of the crystallization interface and directly affects the crystal quality. However, in microgravity environment, the buoyancy convection is almost eliminated, so the crystal growth process approaching to a pure diffusion situation is possible. Therefore, to grow crystal in solution in microgravity environment has been an important research direction for a long time. In this paper, optical interferometry technique, computer technique and image processing technique are combined to form a real-time diagnosis method to research the crystal growth process — the optical interferometry technique.

The optical interferometry technique is a non-contact measuring technique. The changing state of flow field can be observed in real-time using this technique. It is a much better choice to research the growth process of solution crystal and the buoyancy convection phenomenon as well as to investigate the gravity and microgravity effects on this process. This technique is a connection between the crystal growth technique and the fluid computation, and it can provide some experimental data for the theoretical research of fluid mechanics exploring the crystal growth. Therefore, it is an important real-time observation technique to investigate the rules of crystal growth in microgravity environment and guide the space experiments.

For a long time, it has been noted that the environmental

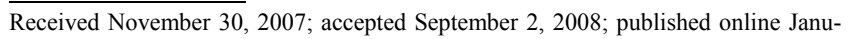
ary 10,2009

doi: $10.1007 / \mathrm{s} 11431-008-0331-2$

†Corresponding author (email: kq@imech.ac.cn)

Supported by the National Natural Science Foundation of China (Grant Nos. 10672171 and 10432060) and Knowledge Innovatiou Program of CAS (Phase III) 
factors have a great effect on the crystal growth process. Some parameters such as concentration and temperature of diffusion layers in the crystal growth process directly influence the crystal growth configuration, quality, etc. Onuma et al. ${ }^{[1]}$ and Miyashita et al. ${ }^{[2]}$ respectively adopted the optical interferometry method to observe the crystal growth, but both of them emphasized their research on the optical experimental technique. Duan, et $\mathrm{al}^{[3]}$ researched the mass transfer process of diffusion in the protein crystal growth and analyzed the diffusion characters of the growth process of protein crystal. Moreover, they also researched the mass transfer process of diffusion between the protein solution and water in microgravity environment and presented the diffusion coefficients of gutta-percha protein solution ${ }^{[4]}$. In this paper, we adopted an optical interference method to observe the growth process of an inorganic salt crystal in real time and used the theoretical idea of fluid mechan$\mathrm{ics}^{[5]}$ to analyze the crystal growth process. This paper explained the convection phenomenon in the growth process of inorganic salt crystal, explored the crystal growth process and analyzed the fluid dynamics mechanism in this process. The experimental method and the research theories in this paper are widely applicable to the analysis and research for the growth process of transparent solution crystal.

\section{Principles}

\subsection{Basic theory of convection}

In the gravity field, the buoyancy convection is a typical fluid mechanics problem that is applied to many engineering problems. The nonuniformity of temperature or concentration leads to the nonuniformity of fluid density, and the fluid with lower density will move to the reverse direction of gravity field because of the buoyancy. When the buoyancy and heat effects are high enough to overcome viscosity resistance, the convection in the fluid will happen, which is natural convection. Usually, Rayleigh number is introduced as the critical parameter. Rayleigh number of concentration convection is defined as

$$
R a=\frac{\rho g \beta_{\mathrm{C}} \Delta C d^{3}}{\mu D},
$$

where $\beta_{\mathrm{C}}, \mu$, and $D$ are solute expansion coefficient, kinetic viscosity coefficient, and solute diffusion coefficient, respectively; $d$ is the character size; $\Delta C$ is the concentration difference. When $R a \approx 1700$, the convec- tion elementary cell will appear, and the corresponding Rayleigh number is the critical Rayleigh number, which is defined as $(R a)_{C}$. Therefore, in the gravity field, the buoyancy convection is a key environmental factor to influence the crystal growth.

In microgravity environment, the value of gravity acceleration $g$ is very small, so Rayleigh number will decrease greatly, thus the buoyancy convection is restrained. At this time, other convection phenomena will be obvious, among which the convection driven by gradient of surface tension is often discussed, i.e., the nonuniformity of surface tension drives the fluid to move from a higher temperature area to a lower temperature area in the surface and also from a higher concentration area to a lower concentration area to form the convection. As long as the nonuniformity of temperature or concentration exists in the surface of the fluid, the fluid will keep moving; when the temperature difference or the concentration difference in the surface of the fluid exceeds the critical value, the thermal capillary convection or concentration capillary convection driven by the surface tension will be changed from steady flow to oscillatory flow. Therefore, in the microgravity environment, the capillary convection becomes the key environmental factor to influence the crystal growth.

\subsection{Basic principle of phase shift}

In this paper, we adopted a four-step phase shift technique to analyze interference fringes and get the physical measurements from the interference fringes. The basic principle of the phase shift is to introduce an assigned phase change into physical light wave or reference light wave (reference light wave in this paper) in order to obtain a changed distribution of light intensity. A phase distribution of the original fringes can be obtained through processing different light intensity distributions obtained after many phase shifts. In this paper, we adopted a four-step phase shift method with equal step length. The step length can be arbitrarily selected between 0 and $\pi / 2$. After four equal-step-length phase shifts continually introduced with the step length $\delta=2 \varepsilon$, the obtained four figures of fringes can be expressed by the following four equations:

$$
\begin{gathered}
A(x, y)=I_{0}\{1+\gamma \cos [\phi(x, y)-3 \varepsilon]\}, \\
B(x, y)=I_{0}\{1+\gamma \cos [\phi(x, y)-\varepsilon]\}, \\
C(x, y)=I_{0}\{1+\gamma \cos [\phi(x, y)+\varepsilon]\},
\end{gathered}
$$




$$
D(x, y)=I_{0}\{1+\gamma \cos [\phi(x, y)+3 \varepsilon]\} .
$$

Solving the set of equations above, we can obtain

$$
\bar{\phi}=\operatorname{tg}^{-1}\left\{\frac{\sqrt{[(A-D)+(B-C)] \cdot[3(B-C)-(A-D)]}}{|(B+C)-(A+D)|}\right\},
$$

where $\phi \in[0, \pi / 2]$. In order to expand the phase easily, it should be expanded to $[-\pi, \pi]$. The expansion method is as follows:

$$
\phi(x, y)= \begin{cases}\bar{\phi}(x, y), & (B-C)>0,(B+C)-(A+D)>0 \\ \pi-\bar{\phi}(x, y), & (B-C)>0,(B+C)-(A+D)<0 \\ -\pi+\bar{\phi}(x, y), & (B-C)<0,(B+C)-(A+D)<0 \\ -\bar{\phi}(x, y), & (B-C)<0,(B+C)-(A+D)>0\end{cases}
$$

\section{Experiments and results}

\subsection{Experiment technique}

A Mach-Zehnder interference system is shown in Figure 1. The laser light transmitted from He-Ne laser is expanded by lens L1 and modulated by lens L 2 to form a parallel light beam, then it is separated into two beams by beam splitter Bs1. One is the physical light wave that is reflected by mirror M1 and passes through the crystal growth cell and the beam splitter Bs2. The other one is the reference light wave that is reflected by mirror M2, installed on the piezoelectric ceramic used for four-step phase shift, and reflected by beam splitter Bs2. Then, the physical light wave and the reference light wave join together to create interference fringes after passing through Bs2. Lenses L3, L4 and CCD compose a full flow field image capturing system, whose real measuring size is $4 \mathrm{~mm} \times 5 \mathrm{~mm}$. According to Mach-Zehnder interference fringes, the distribution of refractive index, which is directly relative to concentration distribution of mother liquid, can be calculated in the crystal growth process.

\subsection{Measurement of relationship between refractive index and concentration}

Only the change of the refractive index can be obtained from the experiment image, while it is directly relative to the concentration in this experiment. In order to obtain the change of concentration, we adopted abbe refractometer to get the relationship between the refractive index $n$ and the concentration $C$ of $\mathrm{NaClO}_{3}$ solution. In this experiment, we used a circulating water system to guarantee that the solution was at a constant temperature in measuring. Therefore, the refractive index changed only with the change of solution concentration. The relation curve between refractive index $n$ and concentration $C$ is depicted in Figure 2, and the relationship is described as

$$
n=1.3385+0.0108 C \text {. }
$$

\subsection{Experiment condition and image collection mode}

$\mathrm{NaClO}_{3}$ solution with $46.43 \%$ concentration was confected at the temperature of $21.0^{\circ} \mathrm{C}$ and injected into a crystal growth cell with a capacity of $20 \mathrm{~mm} \times 12 \mathrm{~mm} \times 14$ mm. A piece of $\mathrm{NaClO}_{3}$ crystal sized $1.80 \mathrm{~mm} \times 5.02$ $\mathrm{mm} \times 0.82 \mathrm{~mm}$ was put into the solution as the seedcrystal. The upper face of the crystal was face [001], and both of the left and right faces were face [010]. The seed-crystal started to dissolute slowly after being put into the solution because the solution was not saturated at the beginning. With the dissolution of the crystal, the temperature of the solution decreased and the solution reached saturation and even supersaturation. Then the crystal started to grow gradually. The temperature of the solution was kept at $17^{\circ} \mathrm{C}-20^{\circ} \mathrm{C}$ when the crystal was growing.

The image collection system adopted Matrox Parlsa image board and the corresponding image collection software to collect the interference fringes into the computer by using the four-step phase shift method with the scheduled collection interval $\Delta t=120 \mathrm{~s}$. The total number of the collected images was 65 frames and the image collection time was $130 \mathrm{~min}$. The growth time of the crystal was $6 \mathrm{~h}$ and $10 \mathrm{~min}$. The distribution of refractive index of the solution in the crystal growth process can be calculated by the four-step phase shift method

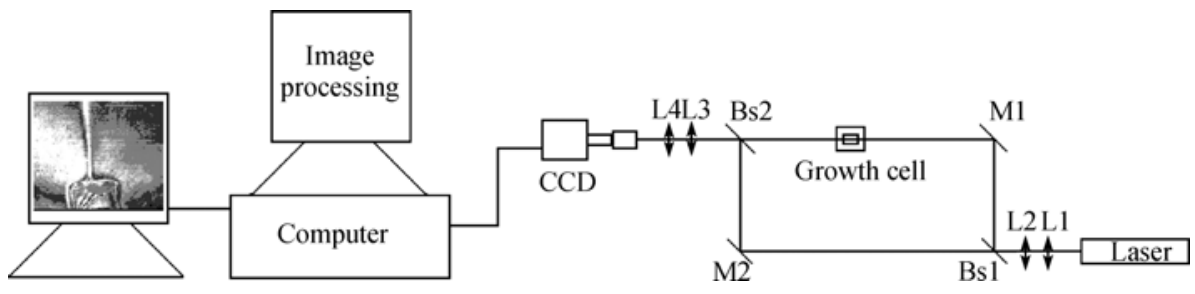

Figure 1 Schematic diagram of optical interference system. 


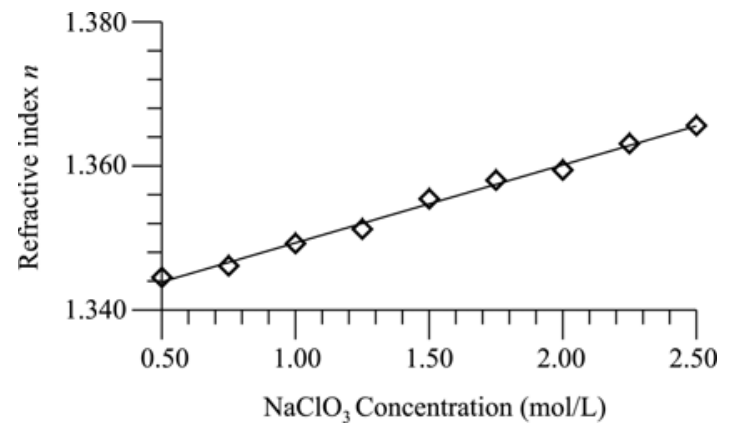

Figure 2 Relation curve between refractive index and concentration.

and the concentration information can be obtained according to the distribution of the refractive index.

\subsection{Experiment results and analyses}

The experiment results are shown in Figure 3. The crystal starts to dissolve after being put into the growth cell because the solution is not saturated at the beginning. The concentration of the solution close to the crystal surface is much higher than that of the solution around due to the dissolution of the crystal. The solution with higher concentration is much heavier and moves downward. This is the buoyancy convection created by the change of concentration of the solution in the gravity field, which is called dissolution vortex. In this experiment, the interference fringes reflect the concentration information. The same interference fringe is in a zone with the same concentration. Therefore, the interference fringes observed in the Mach-Zehnder interference system is around the crystal and downward. The interference fringes and the concentration distribution are depicted in Figure 3(a). When the temperature of the solution decreases to $20^{\circ} \mathrm{C}$, the solution starts to reach the saturated state and the crystal does not dissolve or grow at that time. Because some of the crystal has been dissolved, there is a concentration gradient in the solution and the concentration layer phenomenon appears in the growth cell because of the action of gravity. The interference fringes are horizontal, which are shown in Figure 3 (b). When the temperature continues to decrease to $20^{\circ} \mathrm{C}-17^{\circ} \mathrm{C}$, the solution in the growth cell reaches supersaturation. The crystal at this time is slowly growing and the solute in the solution starts to be transported onto the crystal gradually; the solution close to the crystal surface is lighter with much lower concentration and moves upwards. This fluid flow is also the buoyancy convection created by the change of concentration in the gravity field, which is called growth vortex. The inter- ference fringes observed in the Mach-Zehnder interference system are penniform. Figures 3(c)-3(d) reflect the changing process. The results show that in the crystal growth process, the buoyancy convection caused by the gravity is very clear; this phenomenon can accelerate the exchange of solute so as to affect the growth kinetics of crystal, impurity capture, component uniformity, state stability and so on. The solute demanded for the growth of some crystal faces is insufficient due to the convection, and the supersaturation degree close to these crystal faces is greatly different from those of solution close to other crystal faces, which makes the crystal face fringes darken gradually in the growth process and finally results in that the mother liquid is contained inside the crystal and the layer appears on the crystal. The crystal face containing mother liquid will be in a dislocation state; the energy is much higher and the growth speed is very fast. As a result, each crystal face does not grow up equally due to the convection, which directly influences the growth rate of each crystal face. The size of the crystal becomes $2.44 \mathrm{~mm} \times 1.02 \mathrm{~mm} \times 5.44 \mathrm{~mm}$ after $6 \mathrm{~h}$ and $10 \mathrm{~min}$; the average growth rate of the crystal can be calculated as follows:

$$
\begin{gathered}
U_{(010)}=\frac{(2.44-1.80) \times 10^{-3}}{3600 \times 6+60 \times 10} \times \frac{1}{2}=1.44 \times 10^{-8}, \\
U_{(001)}=\frac{(1.02-0.82) \times 10^{-3}}{3600 \times 6+60 \times 10}=9.0 \times 10^{-9} .
\end{gathered}
$$

Because the buoyancy convection plane is parallel to (010) face and vertical to (001) face, the growth rate of (010) face must be faster than that of (001) face. In addition, the figure of interference fringes also shows that the diffusion layer exists in the crystal growth process. According to the data of concentration change calculated at $t=5400 \mathrm{~s}$ in the experiment, the concentration distributions are given along the straight lines of $y=3.2465$ $\mathrm{mm}$ and $x=2.5592 \mathrm{~mm}$. In Figure 4, the horizontal axis represents the position and the perpendicular axis represents the concentration difference between the local concentration and the boundary concentration of the crystal. The result is depicted in Figure 4. From these two curves, it is shown that the diffusion layer exists obviously in the crystal growth process; the thicknesses of the diffusion layers are $300 \mu \mathrm{m}$ in (010) face and 600 $\mu \mathrm{m}$ in (001) face. For the two crystal faces, the concentration changes inside the diffusion layer are the same as $0.046 \mathrm{~mol} / \mathrm{L}$. However, the concentration gradients are different because of the different thickness of the diffu- 

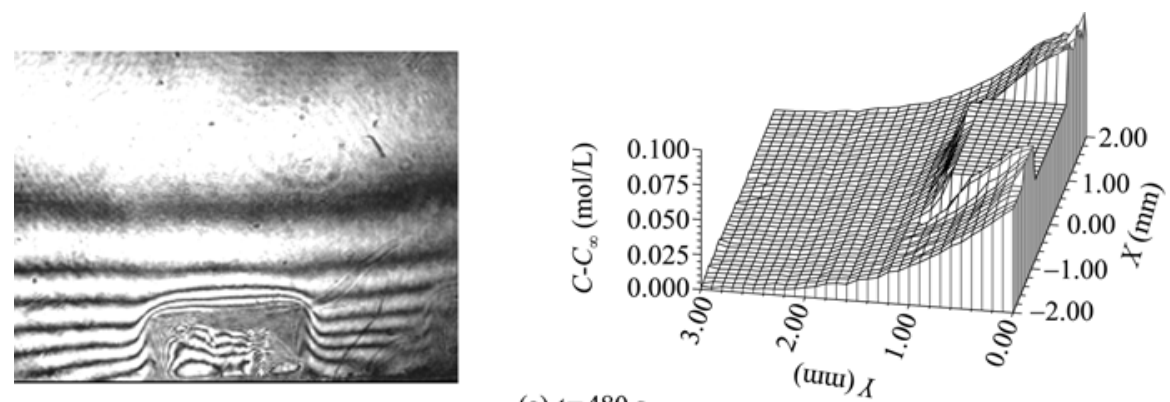

(a) $t=480 \mathrm{~s}$
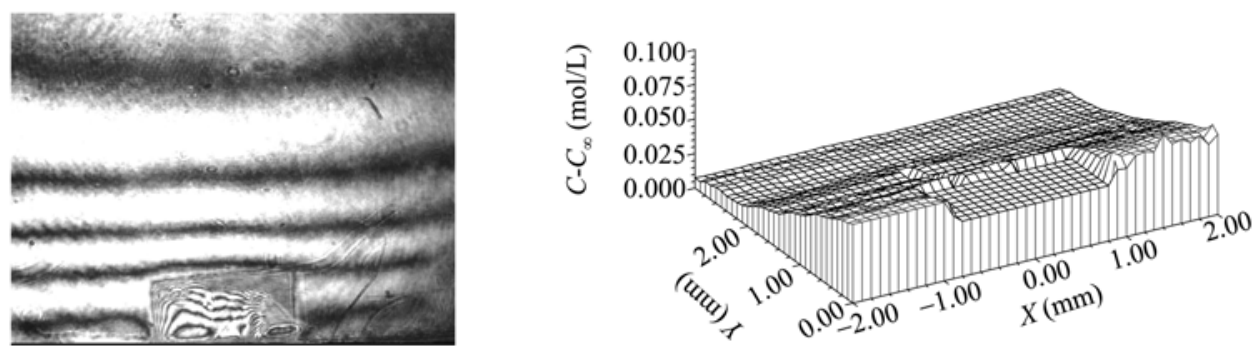

(b) $t=1080 \mathrm{~s}$
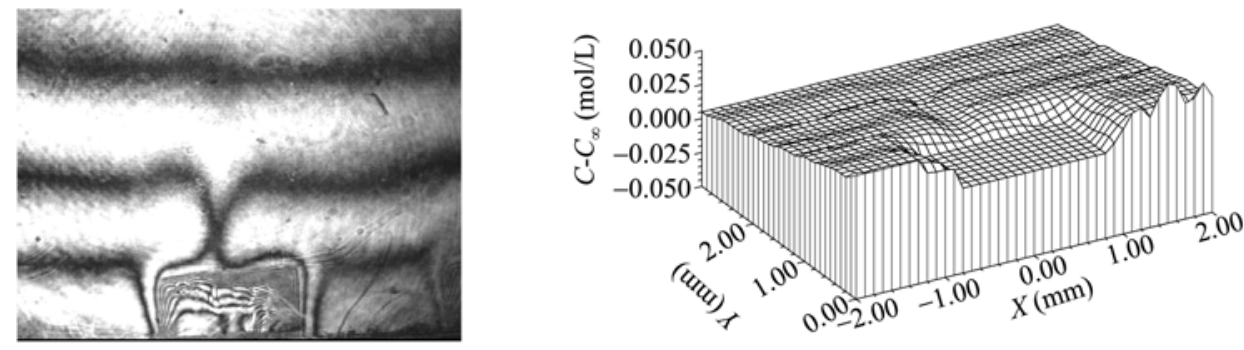

(c) $t=1800 \mathrm{~s}$
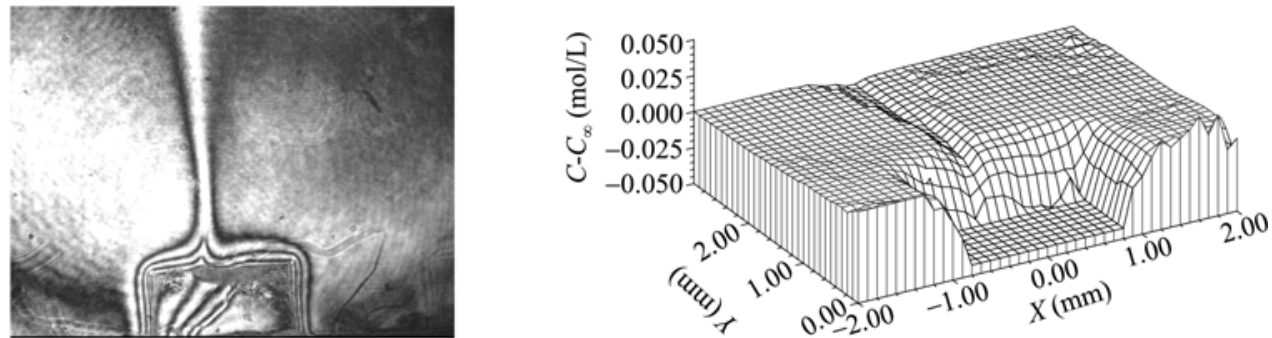

(d) $t=5400 \mathrm{~s}$

Figure 3 Distributions of interference fringes and concentrations in $\mathrm{NaClO}_{3}$ crystal growth process $(5 \mathrm{~mm} \times 4 \mathrm{~mm})$ (the crystal top surface is face [001], and the two side surfaces are face [010]).

sion layers. The gradient in (010) face is much bigger, so the growth rate in (010) face is much faster.

From the experiment results, we can predict that the penniform interference fringes will not be created when growing $\mathrm{NaClO}_{3}$ crystal under microgravity condition because the buoyancy convection is restrained; the interference fringes can only be around the crystal and the crystal will grow up slowly by diffusion, so the growth rate of each crystal face should be the same.

According to hydrokinetics theory, the Rayleigh number reflects whether there is convection or not. When $R a=1700$, the buoyancy convection will appear. In the light of this theory, we can calculate the Rayleigh number for the $\mathrm{NaClO}_{3}$ crystal growth system.

For $\mathrm{NaClO}_{3}$ solution, the solution density $\rho$ is $1.446 \times 10^{3} \mathrm{~kg} / \mathrm{m}^{3}$, the expansion coefficient of solute $\beta_{\mathrm{C}}$ is $5.5 \times 10^{-5} \mathrm{~m}^{3} / \mathrm{mol}$, the kinematic-viscosity coefficient $\mu$ is $1.3 \times 10^{-6} \mathrm{~m}^{2} / \mathrm{s}$, and the diffusion coefficient of solute $D$ is $1.5 \times 10^{-9} \mathrm{~m}^{2} / \mathrm{s}$. The character scale should be the changing range of concentration in the crystal growth 

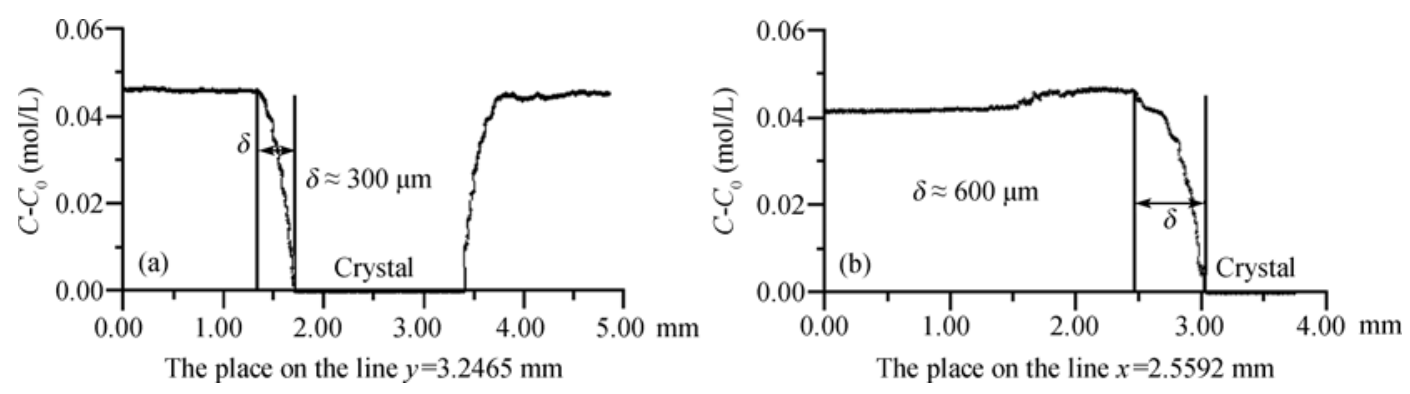

Figure 4 Concentration distributions around the crystal.

process However in this experiment, the concentration change only happens in a thin layer which is called the diffusion layer. Because of the effect of gravity, the thickness of diffusion layer in a different crystal face is a little different. In the growth process of $\mathrm{NaClO}_{3}$ crystal, the thicknesses of diffusion layers are about $300 \mu \mathrm{m}$ in (010) face and about $600 \mu \mathrm{m}$ in (001) face. In this experiment, the buoyancy convection is in (001) face, so the character scale $d$ is selected as about $6.0 \times 10^{-4} \mathrm{~m}$. The concentration difference $\Delta C$ is $0.046 \mathrm{~mol} / \mathrm{L}$. The Rayleigh number is calculated as follows:

$$
\begin{aligned}
R a & =\frac{g \beta_{\mathrm{C}} \Delta C d^{3}}{\mu D} \\
& =\frac{9.8 \times 5.5 \times 10^{-2} \times 0.046 \times\left(6.0 \times 10^{-4}\right)^{3}}{1.3 \times 10^{-6} \times 1.5 \times 10^{-9}}=2746 .
\end{aligned}
$$

The result shows that the buoyancy convection must exist in the growth process of the $\mathrm{NaClO}_{3}$ crystal. The theoretical calculation is consistent with the actual observation.

\section{Conclusions}

This real-time phase shift Mach-Zehnder interference system can observe the concentration change of solution in crystal growth process in real time without disturbance. It is very useful to research the relationship between the fluid flow, characters of heat and mass transfer, and microstructure of the growing crystal. This work has established a research method in crystal growth using theories of fluid mechanics, provided sufficient ground- based experimental preparations for space experiments of crystal growth and supplied reliable experiment measurements. This paper has used Mach-Zehnder interferometry system to observe the dissolution and crystallization processes of $\mathrm{NaClO}_{3}$ crystal in real time and obtained the dissolution fringes and growth fringes of the crystal. The concentration distribution of the solution was also calculated from the interference fringes. In this experiment, the diffusion layer and the buoyancy convection caused by gravity were also observed in the crystal growth process. The buoyancy convection directly influenced the growth rate of each crystal face. In addition, the crystal growth state under microgravity condition was predicted. The experimental method and research theory in this paper are widely applicable to the analysis and research of the growth process of transparent solution crystal.

1 Onuma K, Tsukamoto K, Nakadate S. Application of real time phase shift interferometer to the measurement of concentration field. J Cryst Growth, 1993, 129: 706-718[DOI]

2 Miyashita S, Komatsu H, Suzuki Y, et al. Observation of the concentration distribution around a growing lysozyme crystal. J Cryst Growth 1994, 141: 419-424[DOI]

3 Duan L, Kang Q, Hu W R, et al. Mass transfer process and growth Rate of protein crystal growth. Biophys Chem, 2002, 97: 189-201

4 Duan L, Sun Z W, Kang Q, et al. Observation of diffusion process of a water droplet immersed in protein solution in microgravity. Chin Phys Lett, 2007, 24(12): 3358-3361[DOI]

$5 \mathrm{Hu}$ W R, Xu S C. Microgravity Fluid Mechanics (in Chinese). Beijing: Science Press, 1999 